\title{
Effect of Different Chemicals on the Microbial Growth during Vase Life Period of Cut Rose cv. 'First Red'
}

\author{
V. Vijaya Bhaskar ${ }^{1 *}$, P. Venkata Rao ${ }^{1}$ and R. Subhash Reddy ${ }^{2}$ \\ ${ }^{1}$ Department of Horticulture, College of Agriculture, Acharya N. G. Ranga Agricultural \\ University, Rajendranagar, Hyderabad-500 030, Telangana, India \\ ${ }^{2}$ Department of Microbiology and Bioenergy, College of Agriculture, Acharya N. G. Ranga \\ Agricultural University, Rajendranagar, Hyderabad-500 030, Telangana, India \\ *Corresponding author
}

\begin{tabular}{|c|c|}
\hline \multicolumn{2}{|r|}{ A B S T R A C T } \\
\hline & \multirow{5}{*}{$\begin{array}{l}\text { Chemicals belonging to different groups were employed in the vase water either alone or } \\
\text { in combination to assess their effect on microbial growth during vase life period of cut rose } \\
\text { cv. First Red. The individual chemical treatments SHC } 20 \mathrm{ppm}, \mathrm{Ca}\left(\mathrm{NO}_{3}\right)_{2}, 250 \mathrm{ppm} \text {, AA } \\
150 \mathrm{ppm} \text { have recorded significantly least microbial growth from their respective groups. } \\
\text { Among the Sucrose concentrations Sucrose } 3 \% \text { has recorded significantly highest } \\
\text { microbial growth in the vase solution. Sucrose } 3 \% \text { was combined together with the best } \\
\text { treatments of the above said, as two chemical, three chemical and four chemical } \\
\text { combinations to test their combined effect on microbial growth. Among all the } \\
\text { combinations SHC } 20+\text { Sucrose } 3 \% \text { followed by SHC } 20+\mathrm{Ca}\left(\mathrm{NO}_{3}\right)_{2} 250 \text { and SHC } 20+ \\
\text { AA } 150+\text { Sucrose } 3 \% \text { have inhibited the microbial growth in the vase solution. The } \\
\text { present study suggests that the incorporation of anti-microbial compounds in the vase } \\
\text { solution increased the flower diameter as well as vase life by preventing the proliferation } \\
\text { of microbes in the vase solution. }\end{array}$} \\
\hline & \\
\hline $\begin{array}{l}\text { Micr } \\
\text { Germ } \\
\text { Chen } \\
\text { diam }\end{array}$ & \\
\hline $\mathbf{A r}$ & \\
\hline $\begin{array}{l}\boldsymbol{A} \\
0 \\
\boldsymbol{A}\end{array}$ & \\
\hline
\end{tabular}

\section{Introduction}

Rose ranks first among the cut flowers trade both in the domestic as well as international markets. However, the diminishing keeping quality of cut roses is badly affecting the growers as well as the traders. The most common cause of the termination of vase life in cut roses is water stress (Halevy, 1976). The presence and rapid proliferation of microorganisms in the vase water is thought to result in the occlusion of xylem vessels, water stress and a subsequent reduction in the cut flower longevity (van Doorn and Perik, 1990; van Doorn, 1997).
A variety of germicides have been suggested by several research workers (Aarts, 1957; Marousky, 1969 and 1971; Halevy and Mayak, 1981) to prevent this problem. Many studies have correlated an increase in the bacterial count in vase water with decreased longevity (Larsen and Frolich, 1969; van Doorn and Perik, 1990). However, the response of many cut flowers to germicides is highly variable among species and varieties. Longevity was consistently improved in cut roses with the addition of germicides (Marousky, 1969; van Doorn and Perik, 1990) 
in vase water. Optimal postharvest life of cut flowers is often improved by using suitable vase solutions (Halevy and Mayak, 1979 and 1981). An effective flower food i.e., a preservative solution should contain three basic components to extend the life of cut flowers. A 'Sugar' - to provide energy to the flower, a 'Germicide' - to kill the bacteria and other microorganisms, and an 'Acidifier'- to lower the $\mathrm{pH}$ of water which makes the water wetter there by increases and maintains the uptake of water and nutrients by the flowers (Coake, 1997). Further, many studies tested the effect of commercial preservatives where it is difficult to determine whether any beneficial effect on the flowers resulted from the sugar, the germicide or some other compound contained in the formulation (Staby et al., 1978; Healy and Lang, 1989; Dan and Griffith, 1990).

Results from most studies were found variable and difficult to compare due to lack of consistency in establishing a particular criterion for termination of vase life especially under experimentation conditions. The present study was attempted with the main aim to clarify the effect of different chemicals, either alone or in combination, on the microbial counts found in vase water of cut rose flower. Additionally, assessment of impact of microbes on flower longevity was made.

\section{Materials and Methods}

The present investigation was carried out at Department of Horticulture, College of Agriculture, Acharya N. G. Ranga Agricultural University, Rajendranagar, Hyderabad during the year 2001 and the flowers were held in the laboratory at about $22 \pm 2{ }^{\circ} \mathrm{C}$ room temperature, $50 \%$ to $70 \% \mathrm{RH}$ and $0.38 \mathrm{Wm}^{-2}$ cool-white fluorescent light on a 12 hours photoperiod basis. The aqueous holding solutions used were: Germicides: 8- hydroxy quinoline sulfate (8-HQS) $150 \mathrm{ppm}$ and 300 ppm, Benzalkonium Chloride (BC) $50 \mathrm{ppm}$ and $100 \mathrm{ppm}$, Sodium hypochlorite (SHC) $20 \mathrm{ppm}$ and $40 \mathrm{ppm}$, Control (distilled water); Mineral Salts: Calcium nitrate $\left[\mathrm{Ca}\left(\mathrm{NO}_{3}\right)_{2}\right] 250 \mathrm{ppm}$ and 500 ppm, Aluminum sulfate $\left[\mathrm{Al}_{2}\left(\mathrm{SO}_{4}\right)_{3}\right] 250$ and 500 ppm, Silver thiosulphate (STS) 0.2 and 0.4 $\mathrm{mM}$ and Control (distilled water); Antioxidants: Ascorbic acid (AA) 150 and 300 ppm, Sodium Benzoate (SB) 150 and 300 ppm, Sodium Borohydride (SBH) 150 and $300 \mathrm{ppm}$ and Control (distilled water); Sucrose concentrations: Sucrose 0.5, 1.0, 1.5, 2.0, 2.5, 3.0\% and Control (distilled water); Combinations: $\mathrm{T}_{1}$ : Sodium hypochlorite 20 ppm + Sucrose 3\% (SHC $20+$ Sucrose 3\%), $\mathrm{T}_{2}$ : Ascorbic acid $150 \mathrm{ppm}+$ Sucrose 3\% (AA $150+$ Sucrose 3\%), $\mathrm{T}_{3}$ : Calcium nitrate $250 \mathrm{ppm}+$ Sucrose 3\% $\left(\mathrm{Ca}\left(\mathrm{NO}_{3}\right)_{2} 250+\right.$ Sucrose 3\%), $\mathrm{T}_{4}$ : Sodium hypochlorite 20 ppm + Ascorbic acid 150 ppm (SHC $20+$ AA 150), $\mathrm{T}_{5}$ : Sodium hypochlorite $20 \mathrm{ppm}+$ Calcium nitrate 250 ppm (SHC $20+$ $\left.\mathrm{Ca}\left(\mathrm{NO}_{3}\right)_{2} 250\right), \mathrm{T}_{6}$ : Ascorbic acid $150 \mathrm{ppm}+$ Calcium nitrate 250 ppm (AA $150+$ $\mathrm{Ca}\left(\mathrm{NO}_{3}\right)_{2}$ 250), $\mathrm{T}_{7}$ : Sodium hypochlorite 20 $\mathrm{ppm}+$ Ascorbic acid $150 \mathrm{ppm}+$ Calcium nitrate $250 \mathrm{ppm}$ (SHC 20 + AA $150+$ $\left.\mathrm{Ca}\left(\mathrm{NO}_{3}\right)_{2} 250\right), \mathrm{T}_{8}$ : Sodium hypochlorite 20 ppm + Ascorbic acid $150 \mathrm{ppm}+$ Sucrose 3\% (SHC $20+$ AA $150+$ Sucrose 3\%), $\mathrm{T}_{9}$ : Sodium hypochlorite $20 \mathrm{ppm}+$ Calcium nitrate $250 \mathrm{ppm}+$ Sucrose 3\% (SHC $20+$ $\mathrm{Ca}\left(\mathrm{NO}_{3}\right)_{2} 250+$ Sucrose 3\%), $\mathrm{T}_{10}$ : Ascorbic acid $150 \mathrm{ppm}+$ Calcium nitrate $250 \mathrm{ppm}+$ Sucrose 3\% (AA $150+\mathrm{Ca}\left(\mathrm{NO}_{3}\right)_{2} 250+$ Sucrose 3\%), $\mathrm{T}_{11}$ : Sodium hypochlorite 20 ppm + Ascorbic acid 150 ppm + Calcium nitrate $250 \mathrm{ppm}+$ Sucrose 3\% (SHC $20+$ AA $150+\mathrm{Ca}\left(\mathrm{NO}_{3}\right)_{2} 250+$ Sucrose $\left.3 \%\right), \mathrm{T}_{12}$ : Control (distilled water). All the holding solutions were prepared by dissolving in distilled water. Silver thiosulphate solutions were prepared by mixing silver nitrate and sodium thiosulphate as per the procedure 
described by Veen (1979). The very freshly prepared solutions were used in the experimentation and the vase water was not changed till the end of vase life period. The experiment was conducted in a completely randomized design and replicated thrice with holding method of treatment. The observations were recorded by adopting the following methods.

\section{Microbial counts (cfu)}

Samples of vase water were collected at the beginning of the experiment i.e., just before immersion of flower stalks (on day one) and at the end of the experiment (on the final day) i.e., immediately after disposal of the experiment. After 10 fold serial dilutions, 1 ml samples were plated out on plate count agar medium (Standard Methods Agar of HiMedia) by pour plate method and incubated at $37^{\circ} \mathrm{C}$ for 2 days and colonies formed were counted. Plate with counts between 30 to 300 were taken as reliable and used for calculating the total colony counts, referred as colony forming units (cfu) and was calculated as below

$$
\mathrm{cfu}=\frac{\mathrm{y}}{\mathrm{dx}}
$$

Where,

$y=$ Number of colonies formed

$\mathrm{d}=$ dilution

$\mathrm{x}=$ volume of the sample taken .

\section{Flower diameter (cm) and Vase life (days)}

The whole flower diameter was recorded before senescence using a slide calipers. Flower vase life was defined as the number of days from the start of the experiment until the flower exhibited bent-neck or petal abscission. Day one was considered from the time flowers were placed in the treatment solution. The data were subjected to statistical analysis and least significant difference was used for comparison between the treatments. Prior to analysis, the microbial population of colony forming units was subject to square root transformation.

\section{Results and Discussion}

Changes in the microbial growth significantly differed (Table 1) with different germicide solutions during vase life period of cut rose cv. First Red. On day zero i.e., just before immersion of the cut roses the vase solutions significantly differed in the microbial growth. Significantly maximum microbial growth was observed with control $\left(2.21 \times 10^{3}\right)$, whereas $\mathrm{BC}$ and SHC solutions were absolutely free of microbial growth. The higher concentration of 8-HQS significantly decreased the microbial growth over the lower concentration of 8 HQS. On day 12 i.e., at the end of vase life period, BC treatments significantly increased the growth rate of microbes, whereas, SHC 20 solution recorded significantly lowest $(4.67 \mathrm{x}$ $10^{3}$ ) microbial growth in the vase solution. The treatments 8-HQS 150 and SHC 40 solutions were found better than control, whereas 8-HQS 300 was at par with control. The nascent chlorine released from sodium hypochlorite has checked the growth of microorganisms in the vase solution so that plugging of the conducting tissue was reduced which finally led to increased rate of water uptake there by increased the vase life. The fact that 8-HQS is a useful agent for precipitating many minor elements $(\mathrm{Cu}, \mathrm{Mn}$, $\mathrm{Fe}, \mathrm{Zn})$, suggests that it acted bacteriostatically and fungistatically by precipitating one or several of these elements so that the microorganisms could not use them (Zentmyer, 1943). Further, the activity of 8-HQS, as an inhibitor of micro-organisms, which produces pectolytic enzymes (Parups and Chan, 1973). This has facilitated 
increased vase life due to increased rate of water uptake by decreased blockage of conducting vessels.

During vase life period of cut rose, the microbial growth rate significantly differed (Table 2) with different mineral salt solutions. On day zero, the vase solutions significantly differed with microbial growth rates. Aluminum concentrations recorded significantly lowest microbial growth rate over all the mineral salts. At the end of vase life period i.e., on day $12, \mathrm{Ca}\left(\mathrm{NO}_{3}\right)_{2}$ concentrations followed by STS $0.2 \mathrm{mM}$ recorded significantly lowest microbial growth, whereas, $\mathrm{Al}_{2}\left(\mathrm{SO}_{4}\right)_{3} 500$ recorded significantly highest microbial growth. Aluminum sulfate 250 and STS $0.4 \mathrm{mM}$ were intermediate in reducing microbial growth in the vase solution. STS $0.2 \mathrm{mM}$ was at par with Control (distilled water). Aluminum sulfate acidified ( $\mathrm{pH} 2.8$ ) the holding solution thus reduced bacterial growth in the vase solution at the beginning of the experiment. However, with the passing of time, aluminum sulfate was unable to reduce the mucilageinduced bacterial growth. van Doorn (1998) reported that exacerbation of mucilage effect was probably due to the blockage by aluminum hydroxide precipitate. Flowers held in solutions containing $\mathrm{Ca}\left(\mathrm{NO}_{3}\right)_{2}$ improved the water uptake due to lower activity of microorganisms which prevented the physiological plugging of the conducting vessels. Finally this led to improved flower opening and vase life. Flowers held in STS $0.4 \mathrm{mM}$ experienced early water stress due to photo-oxidation of $\mathrm{Ag}^{+}$salt in the vase solution, which resulted due to formation of black insoluble compounds. But lower concentration had little effect on the formation of insoluble compounds and water stress. Silver ion undoubtedly affects vase life not only by its ethylene-inhibiting action, but also by its bactericidal property (Ohkawa et. al., 1999). Flowers held in control also experienced early water stress that might be due to a disturbance in the transport of water resulting from the plugging of the conducting tissue by microorganisms.

Table.1 Effect of postharvest application of germicides on microbial counts (cfu), flower diameter (cm) and vase life (days) of cut rose cv. 'First Red'

\begin{tabular}{|c|c|c|c|c|c|}
\hline \multirow[b]{2}{*}{ Treatments } & \multicolumn{2}{|c|}{$\begin{array}{l}\text { Microbial counts } \\
\text { on days (cfu) }\end{array}$} & \multirow{2}{*}{$\begin{array}{c}\text { Flower } \\
\text { diameter } \\
(\mathbf{c m})\end{array}$} & \multirow[t]{2}{*}{$\begin{array}{c}\text { Vase life } \\
\text { (days) }\end{array}$} & \multirow{2}{*}{$\begin{array}{c}\% \text { increase/ } \\
\text { decrease in } \\
\text { vase life over } \\
\text { control }\end{array}$} \\
\hline & $\mathbf{0}$ & 12 & & & \\
\hline 8-HQS 150 & $\begin{array}{l}1.92 \times 10^{2} \\
(13.84)^{b}\end{array}$ & $\begin{array}{l}1.47 \times 10^{4} \\
(121.19)^{\mathrm{c}}\end{array}$ & $4.23^{b}$ & $10.27^{\mathrm{a}}$ & 27.26 \\
\hline 8-HQS 300 & $\begin{array}{c}4.6 \times 10^{1} \\
(6.45)^{c}\end{array}$ & $\begin{array}{l}9.67 \times 10^{4} \\
(310.57)^{b}\end{array}$ & $4.80^{\mathrm{a}}$ & $9.13^{b}$ & 13.14 \\
\hline BC 50 & $\begin{array}{c}0.00 \\
(1.00)^{\mathrm{d}}\end{array}$ & $\begin{array}{l}1.66 \times 10^{5} \\
(393.67)^{\mathrm{a}}\end{array}$ & $4.10^{b}$ & $9.27^{b}$ & 14.87 \\
\hline BC 100 & $\begin{array}{c}0.00 \\
(1.00)^{\mathrm{d}}\end{array}$ & $\begin{array}{l}1.86 \times 10^{5} \\
(430.73)^{\mathrm{a}}\end{array}$ & $3.87^{b}$ & $9.53^{b}$ & 18.09 \\
\hline SHC 20 & $\begin{array}{c}0.00 \\
(1.00)^{\mathrm{d}}\end{array}$ & $\begin{array}{c}4.67 \times 10^{3} \\
(66.37)^{\mathrm{d}}\end{array}$ & $5.03^{\mathrm{a}}$ & $10.80^{\mathrm{a}}$ & 33.83 \\
\hline SHC 40 & $\begin{array}{c}0.00 \\
(1.00)^{\mathrm{d}}\end{array}$ & $\begin{array}{l}4.47 \times 10^{4} \\
(208.14)^{c}\end{array}$ & $3.83^{b}$ & $9.13^{b}$ & 13.14 \\
\hline Control & $\begin{array}{l}2.21 \times 10^{3} \\
(46.96)^{\mathrm{a}}\end{array}$ & $\begin{array}{l}9.83 \times 10^{4} \\
(310.32)^{b}\end{array}$ & $3.70^{b}$ & $8.07^{\mathrm{c}}$ & 0.00 \\
\hline F-test & $* *$ & $* *$ & $* *$ & *** & -- \\
\hline SEm \pm & 0.82 & 33.53 & 0.18 & 0.31 & -- \\
\hline CD 5\% & 2.48 & 101.72 & 0.543 & 0.927 & -- \\
\hline
\end{tabular}


Table.2 Effect of postharvest application of mineral salts on microbial counts (cfu), flower diameter (cm) and vase life (days) of cut rose cv. 'First Red'

\begin{tabular}{|c|c|c|c|c|c|}
\hline \multirow{2}{*}{ Treatments } & \multicolumn{2}{|c|}{$\begin{array}{l}\text { Microbial counts } \\
\text { on days (cfu) }\end{array}$} & \multirow{2}{*}{$\begin{array}{l}\text { Flower } \\
\text { diameter } \\
(\mathrm{cm})\end{array}$} & \multirow[t]{2}{*}{$\begin{array}{l}\text { Vase life } \\
\text { (days) }\end{array}$} & \multirow{2}{*}{$\begin{array}{c}\% \text { increase/ } \\
\text { decrease in } \\
\text { vase life over } \\
\text { control }\end{array}$} \\
\hline & 0 & 12 & & & \\
\hline $\mathrm{Ca}\left(\mathrm{NO}_{3}\right)_{2} 250$ & $\begin{array}{c}2.97 \times 10^{3} \\
(54.44)^{\mathrm{a}}\end{array}$ & $\begin{array}{l}5.4 \times 10^{4} \\
(230.66)^{\mathrm{c}}\end{array}$ & $4.83^{\mathrm{a}}$ & $10.87^{\mathrm{a}}$ & 36.39 \\
\hline $\mathrm{Ca}\left(\mathrm{NO}_{3}\right)_{2} 500$ & $\begin{array}{l}2.69 \times 10^{3} \\
(51.86)^{\mathrm{b}}\end{array}$ & $\begin{array}{l}3.8 \times 10^{4} \\
(194.58)^{\mathrm{c}}\end{array}$ & $4.30^{\mathrm{a}}$ & $10.60^{\mathrm{a}}$ & 33.00 \\
\hline $\mathrm{Al}_{2}\left(\mathrm{SO}_{4}\right)_{3} \mathbf{2 5 0}$ & $\begin{array}{l}8.16 \times 10^{2} \\
(28.51)^{\mathrm{c}}\end{array}$ & $\begin{array}{l}1.67 \times 10^{6} \\
(1280.23)^{\mathrm{b}}\end{array}$ & $5.07^{\mathrm{a}}$ & $10.00^{\mathrm{a}}$ & 25.47 \\
\hline $\mathrm{Al}_{2}\left(\mathrm{SO}_{4}\right)_{3} \mathbf{5 0 0}$ & $\begin{array}{l}3.93 \times 10^{2} \\
(19.86)^{\mathrm{d}}\end{array}$ & $\begin{array}{l}2.76 \times 10^{6} \\
(1658.00)^{\mathrm{a}}\end{array}$ & $4.10^{b}$ & $9.73^{b}$ & 22.08 \\
\hline STS $0.2 \mathrm{mM}$ & $\begin{array}{l}2.44 \times 10^{3} \\
(49.36)^{b}\end{array}$ & $\begin{array}{l}1.60 \times 10^{5} \\
(395.66)^{c}\end{array}$ & $4.07^{b}$ & $10.80^{\mathrm{a}}$ & 35.51 \\
\hline STS $0.4 \mathrm{mM}$ & $\begin{array}{c}3.16 \times 10^{3} \\
(56.15)^{\mathrm{a}}\end{array}$ & $\begin{array}{l}1.50 \times 10^{6} \\
(1183.16)^{b}\end{array}$ & $3.23^{\mathrm{b}}$ & $10.20^{\mathrm{a}}$ & 27.98 \\
\hline Control & $\begin{array}{l}2.49 \times 10^{3} \\
(49.84)^{\mathrm{b}}\end{array}$ & $\begin{array}{l}1.16 \times 10^{5} \\
(340.46)^{\mathrm{c}}\end{array}$ & $4.10^{b}$ & $7.97^{\mathrm{c}}$ & 0.00 \\
\hline F-test & $* *$ & $* *$ & $*$ & $* *$ & -- \\
\hline SEm + & 1.39 & 102.47 & 0.30 & 0.33 & -- \\
\hline CD 5\% & 4.23 & 310.83 & 0.904 & 1.011 & -- \\
\hline
\end{tabular}

Table.3 Effect of postharvest application of antioxidants on microbial counts (cfu), flower diameter (cm) and vase life (days) of cut rose cv. 'First Red'

\begin{tabular}{|c|c|c|c|c|c|}
\hline \multirow{2}{*}{ Treatments } & \multicolumn{2}{|c|}{$\begin{array}{l}\text { Microbial counts } \\
\text { on days (cfu) }\end{array}$} & \multirow{2}{*}{$\begin{array}{l}\text { Flower } \\
\text { diameter } \\
(\mathrm{cm})\end{array}$} & \multirow[t]{2}{*}{$\begin{array}{c}\text { Vase life } \\
\text { (days) }\end{array}$} & \multirow{2}{*}{$\begin{array}{l}\% \text { increase/ } \\
\text { decrease in } \\
\text { vase life over } \\
\text { control }\end{array}$} \\
\hline & 0 & 10 & & & \\
\hline AA 150 & $\begin{array}{l}6.48 \times 10^{1} \\
(8.05)^{\mathrm{c}}\end{array}$ & $\begin{array}{l}1.83 \times 10^{5} \\
(425.98)^{\mathrm{c}}\end{array}$ & 4.99 & $9.27^{\mathrm{a}}$ & 16.90 \\
\hline AA 300 & $\begin{array}{c}6.46 \times 10^{1} \\
(8.02)^{\mathrm{c}}\end{array}$ & $\begin{array}{l}6.93 \times 10^{5} \\
(767.05)^{\mathrm{b}}\end{array}$ & 4.50 & $8.47^{b}$ & 6.81 \\
\hline SB 150 & $\begin{array}{c}2.29 \times 10^{3} \\
(47.81)^{\mathrm{a}}\end{array}$ & $\begin{array}{l}4.5 \times 10^{5} \\
(668.23)^{b}\end{array}$ & 4.03 & $8.53^{b}$ & 7.57 \\
\hline SB 300 & $\begin{array}{l}2.48 \times 10^{3} \\
(49.69)^{\mathrm{a}}\end{array}$ & $\begin{array}{l}5.1 \times 10^{5} \\
(707.03)^{b}\end{array}$ & 3.90 & $8.47^{b}$ & 6.81 \\
\hline SBH 150 & $\begin{array}{c}2.64 \times 10^{3} \\
(51.28)^{\mathrm{a}}\end{array}$ & $\begin{array}{l}1.0 \times 10^{6} \\
(996.98)^{\mathrm{a}}\end{array}$ & 3.53 & $6.53^{c}$ & -17.65 \\
\hline SBH 300 & $\begin{array}{l}1.56 \times 10^{3} \\
(37.90)^{\mathrm{b}}\end{array}$ & $\begin{array}{l}1.3 \times 10^{6} \\
(1136.70)^{\mathrm{a}}\end{array}$ & 3.93 & $7.13^{\mathrm{c}}$ & -10.09 \\
\hline Control & $\begin{array}{c}2.15 \times 10^{3} \\
(46.38)^{\mathrm{a}}\end{array}$ & $\begin{array}{l}9.9 \times 10^{5} \\
(988.47)^{\mathrm{a}}\end{array}$ & 3.87 & $7.93^{b}$ & 0.00 \\
\hline F-test & $* *$ & $* *$ & NS & $* *$ & -- \\
\hline SEm \pm & 3.25 & 110.51 & 0.29 & 0.24 & -- \\
\hline CD 5\% & 9.86 & 335.22 & - & 0.717 & -- \\
\hline
\end{tabular}


Table.4 Effect of postharvest application of sucrose concentrations on microbial counts (cfu), flower diameter (cm) and vase life (days) of cut rose cv. 'First Red'

\begin{tabular}{|c|c|c|c|c|c|}
\hline \multirow[t]{2}{*}{ Treatments } & \multicolumn{2}{|c|}{$\begin{array}{l}\text { Microbial counts } \\
\text { on days (cfu) }\end{array}$} & \multirow{2}{*}{$\begin{array}{l}\text { Flower } \\
\text { diameter } \\
(\mathrm{cm})\end{array}$} & \multirow[t]{2}{*}{ Vase life (days) } & \multirow{2}{*}{$\begin{array}{c}\text { \% increase/ decrease } \\
\text { in vase life over } \\
\text { control }\end{array}$} \\
\hline & 0 & 12 & & & \\
\hline Sucrose $0.5 \%$ & $\begin{array}{l}1.62 \times 10^{4} \\
(127.27)^{\mathrm{a}}\end{array}$ & $\begin{array}{l}5.93 \times 10^{5} \\
(751.90)^{b}\end{array}$ & 3.53 & $6.60^{c}$ & -0.09 \\
\hline Sucrose $1.0 \%$ & $\begin{array}{l}1.99 \times 10^{4} \\
(140.89)^{\mathrm{a}}\end{array}$ & $\begin{array}{l}3.29 \times 10^{5} \\
(568.05)^{\mathrm{c}}\end{array}$ & 3.97 & $7.33^{\mathrm{b}}$ & 0.03 \\
\hline Sucrose 1.5\% & $\begin{array}{l}1.95 \times 10^{4} \\
(139.23)^{\mathrm{a}}\end{array}$ & $\begin{array}{l}5.6 \times 10^{5} \\
(725.98)^{b}\end{array}$ & 3.73 & $6.60^{c}$ & -0.09 \\
\hline Sucrose $2.0 \%$ & $\begin{array}{l}1.91 \times 10^{4} \\
(138.07)^{\mathrm{a}}\end{array}$ & $\begin{array}{l}1.28 \times 10^{6} \\
(1128.63)^{b}\end{array}$ & 4.13 & $6.80^{\mathrm{b}}$ & -0.06 \\
\hline Sucrose $2.5 \%$ & $\begin{array}{c}2.13 \times 10^{4} \\
(145.23)^{\mathrm{a}}\end{array}$ & $\begin{array}{l}1.20 \times 10^{6} \\
(1093.92)^{b}\end{array}$ & 4.00 & $7.33^{b}$ & 0.03 \\
\hline Sucrose 3.0\% & $\begin{array}{l}2.08 \times 10^{4} \\
(142.81)^{\mathrm{a}}\end{array}$ & $\begin{array}{c}1.59 \times 10^{7} \\
(3968.47)^{\mathrm{a}}\end{array}$ & 4.47 & $8.13^{\mathrm{a}}$ & 0.12 \\
\hline Control & $\begin{array}{c}1.72 \times 10^{3} \\
(41.51)^{\mathrm{b}}\end{array}$ & $\begin{array}{l}7.60 \times 10^{4} \\
(265.11)^{\mathrm{c}}\end{array}$ & 3.60 & $7.27^{b}$ & 0.00 \\
\hline F-test & $* *$ & $* *$ & NS & $* *$ & -- \\
\hline SEm \pm & 7.82 & 139.88 & 0.230 & 0.210 & -- \\
\hline CD 5\% & 23.72 & 424.34 & -- & 0.626 & -- \\
\hline
\end{tabular}

Table.5 Effect of postharvest application of combinations on microbial counts (cfu), flower diameter (cm) and vase life (days) of cut rose cv. 'First Red'

\begin{tabular}{|c|c|c|c|c|c|}
\hline \multirow[t]{2}{*}{ Treatments } & \multicolumn{2}{|c|}{$\begin{array}{l}\text { Microbial counts } \\
\text { on days (cfu) }\end{array}$} & \multirow{2}{*}{$\begin{array}{c}\text { Flower } \\
\text { diameter } \\
(\mathrm{cm})\end{array}$} & \multirow[t]{2}{*}{$\begin{array}{c}\text { Vase life } \\
\text { (days) }\end{array}$} & \multirow{2}{*}{$\begin{array}{c}\text { \% increase/ } \\
\text { decrease in vase } \\
\text { life over control }\end{array}$} \\
\hline & $\mathbf{0}$ & 14 & & & \\
\hline SHC $20+$ Sucrose $3 \%$ & $\begin{array}{c}0.00 \\
(1.00)^{\mathrm{e}}\end{array}$ & $\begin{array}{l}6.17 \times 10^{4} \\
(246.44)^{c}\end{array}$ & $7.33^{\mathrm{a}}$ & $12.93^{\mathrm{a}}$ & 51.58 \\
\hline AA $150+$ Sucrose $3 \%$ & $\begin{array}{l}1.29 \times 10^{3} \\
(35.47)^{\mathrm{b}}\end{array}$ & $\begin{array}{l}5.45 \times 10^{6} \\
(2059.92)^{\mathrm{c}}\end{array}$ & $5.97^{b}$ & $9.40^{\mathrm{c}}$ & 10.20 \\
\hline $\mathrm{CA}\left(\mathrm{NO}_{3}\right)_{2} 250+$ Sucrose $3 \%$ & $\begin{array}{c}2.27 \times 10^{3} \\
(47.18)^{\mathrm{a}}\end{array}$ & $\begin{array}{c}1.71 \times 10^{8} \\
(13072.89)^{\mathrm{b}}\end{array}$ & $4.47^{\mathrm{d}}$ & $7.87^{\mathrm{c}}$ & -7.74 \\
\hline SHC $20+$ AA 150 & $\begin{array}{c}2.15 \times 10^{3} \\
(46.04)^{\mathrm{a}}\end{array}$ & $\begin{array}{l}5.9 \times 10^{6} \\
(2396.08)^{\mathrm{c}}\end{array}$ & $5.07^{\mathrm{c}}$ & $9.07^{\mathrm{c}}$ & 6.33 \\
\hline $\mathrm{SHC} 20+\mathrm{Ca}\left(\mathrm{NO}_{3}\right)_{2} 250$ & $\begin{array}{l}3.6 \times 10^{1} \\
(6.07)^{\mathrm{e}}\end{array}$ & $\begin{array}{l}4.4 \times 10^{5} \\
(661.71)^{\mathrm{c}}\end{array}$ & $7.13^{\mathrm{a}}$ & $12.33^{\mathrm{a}}$ & 44.55 \\
\hline $\mathrm{AA} 150+\mathrm{Ca}\left(\mathrm{NO}_{3}\right)_{2} 250$ & $\begin{array}{l}3.9 \times 10^{1} \\
(6.31)^{\mathrm{c}}\end{array}$ & $\begin{array}{l}1.09 \times 10^{6} \\
(1042.88)^{\mathrm{c}}\end{array}$ & $6.40^{\mathrm{b}}$ & $11.27^{\mathrm{b}}$ & 32.12 \\
\hline $\mathrm{SHC} 20+\mathrm{AA} 150+\mathrm{CA}\left(\mathrm{NO}_{3}\right)_{2} 250$ & $\begin{array}{l}1.84 \times 10^{2} \\
(13.62)^{\mathrm{d}}\end{array}$ & $\begin{array}{l}3.93 \times 10^{6} \\
(1771.08)^{\mathrm{c}}\end{array}$ & $6.17^{\mathrm{b}}$ & $12.53^{\mathrm{a}}$ & 46.89 \\
\hline SHC $20+$ AA $150+$ Sucrose $3 \%$ & $\begin{array}{l}1.51 \times 10^{3} \\
(38.79)^{\mathrm{b}}\end{array}$ & $\begin{array}{l}3.97 \times 10^{5} \\
(627.67)^{\mathrm{c}}\end{array}$ & $6.77^{\mathrm{a}}$ & $12.33^{\mathrm{a}}$ & 44.55 \\
\hline $\begin{array}{l}\text { SHC } 20+\mathrm{Ca}\left(\mathrm{NO}_{3}\right)_{2} 250+\text { Sucrose } \\
3 \%\end{array}$ & $\begin{array}{l}4.77 \times 10^{2} \\
(21.75)^{\mathrm{c}}\end{array}$ & $\begin{array}{l}9.4 \times 10^{5} \\
(968.54)^{\mathrm{c}}\end{array}$ & $5.83^{b}$ & $8.87^{\mathrm{c}}$ & 3.99 \\
\hline $\begin{array}{l}\text { AA } 150+\mathrm{Ca}\left(\mathrm{NO}_{3}\right)_{2} 250+\text { Sucrose } \\
3 \%\end{array}$ & $\begin{array}{l}4.67 \times 10^{2} \\
(21.59)^{c}\end{array}$ & $\begin{array}{l}2.62 \times 10^{7} \\
(4760.79)^{c}\end{array}$ & $5.57^{\mathrm{c}}$ & $8.20^{c}$ & -3.87 \\
\hline $\begin{array}{l}\text { SHC } 20+\mathrm{AA} 150+\mathrm{Ca}\left(\mathrm{NO}_{3}\right)_{2} 250+ \\
\text { Sucrose } 3 \%\end{array}$ & $\begin{array}{l}1.52 \times 10^{3} \\
(38.97)^{\mathrm{b}}\end{array}$ & $\begin{array}{l}4.81 \times 10^{8} \\
(20499.50)^{\mathrm{a}}\end{array}$ & $4.87^{\mathrm{d}}$ & $7.13^{\mathrm{d}}$ & -16.41 \\
\hline Control (distilled water) & $\begin{array}{l}1.88 \times 10^{3} \\
(43.23)^{\mathrm{a}}\end{array}$ & $\begin{array}{l}5.72 \times 10^{6} \\
(2245.58)^{\mathrm{c}}\end{array}$ & $4.40^{\mathrm{d}}$ & $8.53^{\mathrm{c}}$ & 0.00 \\
\hline F-test & $* *$ & $* *$ & $* *$ & $* *$ & -- \\
\hline SEm + & 2.30 & 1678.27 & 0.24 & 0.42 & -- \\
\hline CD 5\% & 6.71 & 4898.76 & 0.69 & 1.22 & -- \\
\hline
\end{tabular}

** Significant at $(\mathrm{P} \leq 0.01) \quad *$ significant at $(\mathrm{P} \leq 0.05) \quad$ NS: Not significant 
Significant differences were observed in the microbial activities of antioxidant solutions (Table 3) during the vase life period of cut rose cv. First Red. On day zero, among all the antioxidant solutions flowers treated with AA significantly decreased the microbial growth, whereas, SB and the lower concentration of SBH had no significant differences with control. However, the higher concentration of $\mathrm{SBH}$ was found intermediate in reducing the microbial growth. On day 10, AA 150 recorded significantly lowest microbial growth, whereas, SBH and control recorded significantly higher microbial growth rate in the vase solution. The treatments $\mathrm{SB}$ and AA 300 were found intermediate. Addition of ascorbic acid has checked the growth of microorganisms by acidifying the vase solution and thus extended the longevity of cut roses, which was related to the promotion of water uptake in plant tissues. Further, ascorbic acid being an antioxidant prevented the peroxidation of lipids (data not shown) and thus maintained the integrity of cell membranes (Parups and Chan, 1973).

The changes in microbial counts during vase life period of cut rose cv. First Red held in various Sucrose concentrations were presented in Table 4. On day zero, control (distilled water) recorded significantly lowest number of microbial counts, whereas, there were no significant differences among sucrose concentrations. On day 12 also, control recorded significantly lowest number of microbial counts when compared with all other sucrose concentrations. Flowers treated with Sucrose 3\% recorded significantly highest number of microbial counts when compared with all other treatments. At the end of vase life period the microbial growth was found significantly highest with the sucrose solutions when compared with control. From this it is apparent that the stem xylem vessels rapidly have become occluded by microorganisms and have prevented further entry of water and the dissolved sugar in to the stem.

The changes in microbial counts significantly differed (Table 5) with different holding combinations during the vase life period of cut rose cv. First Red. On day zero, when SHC 20 was used in two chemical combinations, SHC 20 with AA 150 significantly increased microbial growth when compared with other combinations of SHC 20. When three compounds were used together, SHC 20 with AA $150+\mathrm{Ca}\left(\mathrm{NO}_{3}\right)_{2}$ 250 recorded significantly lowest microbial growth over the other three chemical combinations of SHC 20. When AA 150 was used in two chemical combinations, AA150 with $\mathrm{Ca}\left(\mathrm{NO}_{3}\right)_{2} 250$ recorded significantly lowest microbial growth over the other two chemical combinations of AA150. When three chemicals were used together, AA150 with SHC $20+\mathrm{Ca}\left(\mathrm{NO}_{3}\right)_{2} 250$ recorded significantly lowest microbial growth over the other three chemical combinations of AA 150. When $\mathrm{Ca}\left(\mathrm{NO}_{3}\right)_{2} 250$ was used in two chemical combinations, $\mathrm{Ca}\left(\mathrm{NO}_{3}\right)_{2} 250$ with Sucrose $3 \%$ recorded significantly highest microbial growth when compared with other combinations of $\mathrm{Ca}\left(\mathrm{NO}_{3}\right)_{2} 250$.

When three chemicals were used together, $\mathrm{Ca}\left(\mathrm{NO}_{3}\right)_{2} 250$ with SHC $20+\mathrm{AA} 150$ recorded significantly lower microbial growth over the other combinations of $\mathrm{Ca}\left(\mathrm{NO}_{3}\right)_{2} 250$. When Sucrose 3\% was used in two chemical combinations, Sucrose 3\% with SHC 20 recorded absolutely free of microorganisms, whereas Sucrose $3 \%$ with $\mathrm{Ca}\left(\mathrm{NO}_{3}\right)_{2} \quad 250$ recorded significantly highest microbial growth. When Sucrose 3\% was used in three chemical combinations, Sucrose 3\% with SHC $20+$ AA 150 recorded significantly lowest microbial growth over the other combinations of Sucrose 3\%. Control recorded significantly higher microbial growth which was at par with $\mathrm{Ca}\left(\mathrm{NO}_{3}\right)_{2} 250+$ 
Sucrose 3\%. On day 14, the four chemical combination recorded significantly higher microbial growth when compared with all other combinations, followed by $\mathrm{Ca}\left(\mathrm{NO}_{3}\right)_{2}$ $250+$ Sucrose $3 \%$, whereas, there were no significant differences in the remaining combinations along with control. However, SHC $20+$ Sucrose 3\% recorded significantly lowest microbial growth when compared with all other treatments. When $\mathrm{Ca}\left(\mathrm{NO}_{3}\right)_{2} 250+$ Sucrose 3\% was added in the solution with any other chemical either in three combination or four combination, the vase solutions have become turbid with the passing of time.

Further, it significantly reduced the shelf life of cut roses. Mixing the calcium nitrate (nitrogenous compound) with sugar in a holding solution promoted the growth of microorganisms, thus making it more difficult to control the microorganisms than in solutions containing sugar alone. Staden (1974) reported similar observations in cut flowers. SHC 20 with any other compound in three combination without $\mathrm{Ca}\left(\mathrm{NO}_{3}\right)_{2} 250+$ Sucrose $3 \%$ increased the shelf life of cut roses. All the combinations, except $\mathrm{Ca}\left(\mathrm{NO}_{3}\right)_{2}$ 250 along with Sucrose $3 \%$ in any combination significantly improved the flower opening, diameter and vase life when compared with control.

The flower diameter as well as vase life was maximum (7.33 and 12.93 respectively) with SHC $20+$ Sucrose $3 \%$ followed by SHC $20+$ $\mathrm{Ca}\left(\mathrm{NO}_{3}\right)_{2} 250$ and SHC $20+\mathrm{AA} 150+$ Sucrose 3\%. Maintenance of improved water status seems to be the most important aspect in extension of flower longevity. The increased shelf life of flower by SHC $20+$ Sucrose $3 \%$ is an expression of the maintenance of better tissue water potential due to improved water relations in the floral tissue through inhibition of microbial growth in the vase solution by applying SHC 20, the anti-microbial compound. van Doorn et al., (1991) also reported similar observation in cut flowers. When sucrose was given without an anti-microbial compound, microorganisms proliferated rapidly and occluded the xylem vessels by preventing further entry of water and the dissolved sugar.

However, sucrose applied in the vase solution along with other compounds increased the vase life, perhaps by increasing the pool of dry matter and respirable substrate, lowering the osmotic potential of petals (Halevy and Mayak, 1979), promoting respiration (Coorts, 1973) also might have delayed the auto catalytic rise in ethylene production, thus extending the longevity. The extended longevity of $\mathrm{SHC} 20+\mathrm{Ca}\left(\mathrm{NO}_{3}\right)_{2} 250$ was mainly due to their effect of preventing the vascular blockage of the stem and partial substitution for metabolic sugars by calcium nitrate (Halevy, 1976; Mayak et al., 1978).

\section{References}

Aarts, J. F. T. H., 1957. Over de houdbaarheid van snijbloemen (On the keepability of cut flowers). Meded van de Landbouwhoge school te Wageningen 57: 1-62.

Coake, D. L., 1997. Preservatives: A comprehensive guide to fresh flower care and handling products. The American Rose 34: $15-17$.

Coorts, G.D., 1973. Internal metabolic changes in cut flowers. Hort Science 8: 195-198.

Dan, A.J., and Griffith, L.P. 1990. Effects of commercial floral preservatives on four types of cut flowers. Proceedings of Flarida State Horticultural Society 103: 214-217.

Halevy, A. H., and Mayak, S. 1981. Senescence and postharvest physiology of cut flowers Part 2 pp 59-143. In: Horticultural Reviews 3 (ed J Janick) Avi Publishing Company Inc, West Port, Connecticut, USA.

Halevy, A.H., 1976. Treatments to improve water balance of cut flowers. Acta Horticulturae 64: 1223-1230. 
Halevy, A.H., and Mayak, S. 1979. Senescence and postharvest physiology of cut flowers, Part 1 pp 204-236. In: Horticultural Reviews 1 (ed. J Janick). Avi Publishing Company Inc., West Port, Connecticut, USA.

Healy, W., and Lang, D. 1989. Postharvest handling of Alstroemeria. HortScience 24: 641-643.

Larsen, F. E., and Frolich, M. 1969. The influence of 8-hydroxyquinoline citrate, Ndimethylamine succinamic acid, and sucrose on respiration and water flow in 'Red Sim' carnations in relation to flower senescence. Journal of American Society for Horticultural Science 94: 289-292.

Marousky, F. J., 1969. Vascular blockage, water absorption, stomatal opening and respiration of cut 'Better Times' roses treated with 8-hydroxy quinoline citrate and sucrose. Journal of American Society for Horticultural Science 94: 223-226.

Marousky, F. J., 1971. Inhibition of vascular blockage and increased moisture retention in cut roses induced by $\mathrm{pH}, 8$ hydroxyquinoline citrate and sucrose. Journal of American Society for Horticultural Science 96: 38-41.

Mayak, S., Kofranek, A.M. and Tirosh, T. 1978. The effect of inorganic salts on the senescence of Dianthus caryophyllus flowers. Physiologia Plantarum 43: 282286.

Ohkawa, K., Kasahara, Y. and Suh, J.N. 1999. Mobility and effects on vase life of silver containing compounds in cut rose flowers. HortScience 34: 112-113.

Parups, E.V., and Chan, A.P. 1973. Extension of vase life of cut flowers by use of isoascorbate containing preservative solutions. Journal of American Society for Horticultural Science 98: 22-26.

Staby, G.L., Evin T. D. and De Hertogh, A.A. 1978. Cut flower life of tulips as influenced by preservative and water quality. Floricultural Reviews 162(4200): 22-23.

Staden, O.L., 1974. Vase life of cut flowers. Annual Report of Springer Institute 1973. pp 50, Wageningen, Netherlands.

van Doorn, W. G., 1997. Water relations of cut flowers. pp 1-85. In: Horticultural Reviews 18 (ed J. Janick) John Wiley \& Sons Inc, New York, USA.

van Doorn, W. G., and Perik, R. J. J. 1990. Hydroxy quinoline citrate and low $\mathrm{pH}$ prevent vascular blockage in stems of cut rose flowers by reducing the number of bacteria. Journal of American Society for Horticultural Science 115: 979-981.

van Doorn, W.G., 1998. Effects of Daffodil flowers on the water relations and vase life of roses and tulips. Journal of American Society for Horticultural Science 123: 146149.

van Doorn, W.G., de Stigter, H.C.M., de Witte, Y. and Boekestein, A. 1991. Microorganisms at the cut surface and in xylem vessels of rose stems: a scanning electron microscope study. Journal of Applied Bacteriology 70: $34-39$.

Veen, H., 1979. Effects of silver salts on ethylene production and respiration of cut carnations. Acta Horticulturae 91: 99-103.

Zentmyer, G.A., 1943. Mechanism of action of 8hydroxy quinoline. Phytopathology 33: 1121.

\section{How to cite this article:}

Vijaya Bhaskar, V., P. Venkata Rao and Subhash Reddy, R. 2017. Effect of Different Chemicals on the Microbial Growth during Vase Life Period of Cut Rose cv. 'First Red'. Int.J.Curr.Microbiol.App.Sci. 6(10): 812-820. doi: https://doi.org/10.20546/ijcmas.2017.610.097 